\title{
Fatal case of echovirus type 9 encephalitis
}

\author{
M A Zuckerman, M Sheaff, J E Martin, C M Gabriel
}

\begin{abstract}
Enteroviruses are rare causes of acute focal encephalitis. A fatal case of echovirus type 9 infection is reported in a 9 month old boy who presented with a fever and a macular rash followed by two focal seizures. Echovirus type 9 was isolated from lung tissue after seven days.
\end{abstract}

(F Clin Pathol 1993;46:865-866)

The non-polio enteroviruses, in particular about $80 \%$ of the coxsackie virus and echovirus serotypes, are the best known viral associations of aseptic meningitis. They are also rare causes of acute focal encephalitis in children and adolescents. ${ }^{1}$ Echoviruses were noted to be a frequent cause of febrile illness and aseptic meningitis soon after they were first isolated from the faeces of asymptomatic children in the 1950 s.

The clinical features of echoviral infections include a pink macular or maculopapular rash over most of the body which is commonly seen in young children and may last for three to five days. Less often the rash may be petechial or purpuric. In severe infections hepatoadrenal failure has been reported, and in infants with echoviral meningoencephalitis the heart and lungs have also been reported to be affected. Usually the normal course is one of rapid spontaneous clinical improvement. The clinical signs and pathology observed in enteroviral meningoencephalitis vary in severity depending on the extent to which the central nervous system is affected.

Division of Virology,

University College and

Middlesex School of

Medicine, London

M A Zuckerman

Department of

Morbid Anatomy,

The Royal London

Hospital NHS Trust,

Whitechapel, London

M Sheaff

J E Martin

Department of

Peadiatrics, St Albans

City Hospital

C M Gabriel

Correspondence to:

Dr M A Zuckerman

Division of Virology,

University College and

Middlesex School of

Medicine, Windeyer

Building, 46 Cleveland

Street, London W1P 6DB.

Accepted for publication

17 March 1993

\section{Case report}

The patient was one of twins born at 30 weeks' gestation by emergency caesarian section. Progress up to 9 months of age was uneventful until he developed an upper respiratory tract infection together with vomiting and diarrhoea. The rest of the family which included the twin brother and siblings were well. Following this he became feverish, dyspnoeic, and developed a macular rash over his trunk and limbs. The next day he had two focal seizures and was taken to the local casualty department. On examination the macular rash was still present and he had a rectal temperature of $39^{\circ} \mathrm{C}$, central cyanosis, and was in respiratory distress.

Although treatment with intravenous benzylpenicillin and chloramphenicol was started immediately, he sustained a fatal cardiorespi- ratory arrest within a few hours of admission. Investigations, including a lumbar puncture, were not performed due to the patient's rapid clinical deterioration.

\section{Post mortem findings}

External examination showed a fine purpuric rash over the abdomen and limbs, with petechiae over the conjunctiva. Internal examination showed "dull" meninges without an apparent purulent exudate. The gyri were flattened, suggesting raised intracranial pressure, but there was no tonsillar herniation. A petechial rash was seen over the pericardium and thymus and the lung bases were intensely congested. The mesenteric glands were enlarged, the spleen was soft, and both adrenals were dark red.

Histological examination showed congested meningeal vessels with adjacent ischaemic neuronal death and mild perivascular inflammation. No focal pathological changes were observed in the brain. Microscopic examination confirmed the congestion within many of the organs and infiltration of the lungs by lymphocytes, macrophages, and occasional polymorphs. This was consistent with a viral rather than a bacterial infection. There was also bilateral adrenal haemorrhagic necrosis.

Swabs were taken from the following sites-lungs and bronchi, base of brain, meninges, lumbar spine, left and right middle ear and skin. A cisterna magna puncture was performed. No growth was obtained from the meninges, cisterna magna, nor middle ear samples. Skin flora were isolated from the other sites.

Blood cultures were taken on admission
Lung tissue was sent for viral isolation and echovirus type 9 was grown after seven days on MRC-5 tissue culture cells. Unfortunately, no brain tissue was sent for virological analysis.

\section{Discussion}

Herpes simplex virus encephalitis is the most common cause of acute focal encephalitis, and in one series the presumptive diagnosis was confirmed in 194 of 432 patients who underwent brain biopsy. Nine per cent of the rest of the patients with encephalitis had a different viral aetiology and three cases were due to echoviral infection. ${ }^{2}$ Prospective studies of encephalitis diagnosed by brain biopsy 
have shown that Coxsackie virus infections occasionally mimic the clinical features of herpes simplex virus encephalitis. ${ }^{3}$

Infants, especially neonates, are most susceptible to the more serious consequences of enteroviral infections. In a survey of serious echoviral infections in neonates ${ }^{4}$ the most often reported clinical feature of this disease was a severe systemic illness similar to bacterial septicaemia in which hepatic necrosis and haemorrhage were predominant.

In most cases causality is implied by viral isolation from a site other than the central nervous system, a consequence of the difficulty in culturing these viruses from cerebrospinal fluid or brain tissue. These aspects are highlighted in a Canadian survey of enteroviral infections of the central nervous system between $1973-1981^{5}$ in which 12 out of 223 patients who were admitted to hospital presented with encephalitic features. Nine of these patients were aged between 2 months and 7 years. Coxsackie viruses B2, B3, and B5 were isolated from six patients, echoviruses types 3,18 , and 22 from five, and there was one isolate of poliovirus type 2 from a hypogammaglobulinaemic patient. Eight of these isolates were from sites other than the central nervous system. Children or adolescents comprise the seven other reported cases with focal enteroviral encephalitis. ${ }^{1}$

The clinical picture in this case was one of an overwhelming echoviral infection. Focal encephalitis is a rare condition associated with enteroviral infections and this might be because of underreporting due to the problems associated both with viral isolation and also in obtaining samples from patients who spontaneously improve. The use of sensitive diagnostic molecular techniques will allow enteroviral RNA to be detected in cerebrospinal fluid or brain tissue, having reverse transcribed the viral RNA, followed by amplification of the complementary DNA using polymerase chain reaction (PCR) technology. This would allow a better understanding of the true impact of enteroviral infections of the central nervous system. ${ }^{6}$ This may be of importance in the cases which do not spontaneously recover and in whom neurological sequelae may occur. Sells et $a l^{l}$ reported a retrospective survey of 105 patients from whom enteroviruses were isolated from the cerebrospinal fluid or sites other than the central nervous system between 1966-72. Forty of the cases were children, and of the 19 avail- able for follow up, the initial diagnosis in nine was aseptic meningitis; a further nine had a meningoencephalitis; and there was one patient with acute cerebellar ataxia. On full neurological assessment, eight of the 19 still had detectable abnormalities after 17-67 months of follow up.

There is no specific treatment for enteroviral infections but there is some evidence that cell to cell spread of enteroviruses may be minimised by specific neutralising antibody. This approach was based on the fact that serum antibody titres in neonates would be low, and there are reports in which high titre human specific immunoglobulin has been used to treat echoviral encephalitis in children with X-linked agammaglobulinaemia. Intravenous, intrathecal, and intraventricular routes of administration have been tried and varying degrees of success reported. .10 $^{8-10}$ Several antiviral compounds are under evaluation but as yet none has been tested in humans.

Finally, the clinical features of this case highlight the difficulty in differentiating between bacterial septicaemia with or without meningitis and a virological aetiology in this setting.

Dr M A Zuckerman is an MRC Training Fellow. Dr J E Martin is a Wellcome Trust Fellow. We thank Dr Martin is a Wellcome Trust Fellow. We thank Dr isolation and permission to report this case. We thank Ms M Waddle for preparing the typescript

1 Modlin JF, Dagan R, Berlin LE, et al. Focal encephalitis with enterovirus infections. Paediatrics 1991;88:841-5.

2 Whitley RJ, Cobbs CG, Alford CA, et al. Diseases that mimic herpes simplex encephalitis. $\mathcal{F} A M A$ 1989;262: 234-9.

3 Whitley RJ, Soong S-J, Hirsch MS, et al. Herpes simplex encephalitis: Vidarabine therapy and diagnostic probencephalitis: Vidarabine therapy
lems. $N$ Engl $₹$ Med 1981;304:313.

4 Modlin JF. Perinatal echovirus infection: insights from literature review of 61 cases of serious infection and 16 outbreaks in nurseries. Ref Infect Dis 1986;8:918-26.

5 Thiviérge B, Delage G. Infections of the central nervous system caused by enteroviruses: 223 cases seen at a paediatric hospital between 1973-81. Can Med Assoc $f$ 1982;127:1097-102.

6 Rotbart HA. Diagnosis of enteroviral meningitis with the polymerase chain reaction. $¥$ Paediatr $1990 ; 117: 85-9$.

7 Sells CJ, Carpenter RL, Ray CG. Sequelae of central nervous system enterovirus infections. $N$ Engl F Med 1975; 293:1-4.

8 Erlendsson K, Swartz T, Daryer JM. Successful reversal of echovirus encephalitis in X-linked hypogammaglobulinaemia by intraventricular administration of immunoglobulin. $N$ Engl $f$ Med 1985;312:351-3.

9 Johnson PR, Edwards KM, Wright PF. Failure of intraventricular gammaglobulin to eradicate echovirus ventricular gammaglobulin to eradicate echovirus encephalitis in a patient with X-linked a

10 Dwyer JK. Intraventricular gammaglobulin for the management of enterovirus encephalitis. Paed Infect Dis $\mathcal{F}$ 1988;7:530-3. 